\title{
Continuous Noninvasive Ventilation Delivered by a Novel Total Face Mask: A Case Series Report
}

\author{
Inês Belchior MD, Miguel R Gonçalves PT PhD, and João Carlos Winck MD PhD
}

\begin{abstract}
Noninvasive ventilation (NIV) has been widely used to decrease the complications associated with tracheal intubation in mechanically ventilated patients. However, nasal ulcerations may occur when conventional masks are used for continuous ventilation. A total face mask, which has no contact with the more sensitive areas of the face, is a possible option. We describe 3 patients with acute respiratory failure due to amyotrophic lateral sclerosis, who developed nasal bridge skin necrosis during continuous NIV, and one patient with post-extubation respiratory failure due to a high spinal cord injury, who had facial trauma with contraindication for conventional mask use. The total face mask was very well tolerated by all the patients, and permitted safe and efficient continuous NIV for several days until the acute respiratory failure episode resolved. None of the patients required endotracheal intubation during the acute episode. Key words: noninvasive ventilation; interface; total face mask; acute respiratory failure; nasal bridge sore; amyotrophic lateral sclerosis. [Respir Care 2012;57(3):449-453. (C) 2012 Daedalus Enterprises]
\end{abstract}

\section{Introduction}

Noninvasive ventilation (NIV) is the delivery of mechanical ventilation to patients with respiratory failure without the requirement of an invasive artificial airway. Keys to the success of NIV for improving clinical outcomes of patients with acute respiratory failure are careful patient selection and a well designed clinical protocol in order to avoid hindering a potentially more definitive therapy with invasive ventilation. ${ }^{1}$

Apart from the choice of ventilator type, mode, and setting, another crucial issue when starting NIV is to find an optimal interface, since NIV success is highly dependent on the efficacy and comfort of the interface. ${ }^{2}$ It must

The authors are affiliated with the Lung Function and Ventilation Unit, Pulmonology Department, University Hospital of São João, Porto, Portugal.

Dr Belchior has disclosed no conflicts of interest. Dr Winck and Dr Gonçalves have both disclosed a relationship with Philips Respironics.

Correspondence: Inês Belchior MD, Lung Function and Ventilation Unit, Pulmonary Medicine Department, University Hospital of São João, Alameda do Professor Hernâni Monteiro, Porto, Portugal. E-mail: inesbelchior@gmail.com.

DOI: $10.4187 /$ respcare. 01275 assure enough air seal to achieve the warranted ventilatory support, without excessive pressure on the points where it contacts the patient's skin, as to prevent pain and skin sores. ${ }^{2}$ The most common sites of friction and skin damage are the bridge of the nose, the upper lip, and the nasal mucosa. ${ }^{3}$ Gregoretti et $\mathrm{al}^{3}$ have proposed a skin breakdown score (from $0=$ nil, $1=$ area of redness, $2=\bmod$ erate skin breakdown, $3=$ skin ulcer, $4=$ skin necrosis) to quantify the degree of skin lesions during NIV. The most important strategy to prevent skin damage is to avoid an excessively tight fit. Rotating interfaces might reduce the risk of skin damage by changing the distribution of pressure and friction, especially on the bridge of the nose. ${ }^{4,5}$

The most widely used oronasal masks present a discomfort incidence of $30-50 \% .{ }^{4}$ Nasal skin breakdown is the more serious complication and is reported in $5-20 \%$ of cases. ${ }^{6}$ Another limitation for their use is in patients with facial bone fractures or facial abnormalities. ${ }^{7}$

A total face mask has been previously described to improve patient comfort, reduce air leaks, and enhance alveolar ventilation when used in COPD and chest wall disease patients with acute or acute-on-chronic respiratory failure. ${ }^{8} 9$ Problems encountered were secretion encumbrance, air leaks, and aerophagia. The helmet has also been described as an effective interface to deliver NIV in patients with acute respiratory failure, avoiding skin breakdown and optimizing comfort in hypoxemic patients ${ }^{10,11}$ and also 


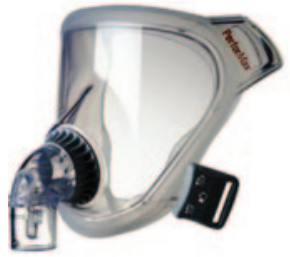

Fig. 1. PerforMax total face mask: lateral view.

in patients with neuromuscular disease. ${ }^{12}$ However, data suggest that NIV delivered by helmet may worsen the patient-ventilator interaction, compared with standard masks, eventually leading to NIV failure. ${ }^{13,14}$ A new, smaller model of a total face mask that covers the entire face (PerforMax, Philips Respironics, Murrysville, Pennsylvania), attempts to overcome these obstacles and permit a safe and efficient NIV application with avoidance of skin breakdown (Fig. 1).

We describe a series of 4 cases of NIV use in neuromuscular and spinal cord lesion patients for distinct purposes, in which patients could not tolerate nasal or oronasal masks and were successfully adapted to the PerforMax total face mask.

\section{Case 1}

A 69-year-old female initiated bi-level NIV (Vivo 40, Breas Medical, Mölnlycke, Sweden) with oxygen supplementation (Table 1) through oronasal mask in the emergency department, due to respiratory acidosis. This treatment improved respiratory failure and hypercapnic encephalopathy. There were no clinical, radiologic, or analytical signs of respiratory infection. The patient's FVC was $520 \mathrm{~mL}$ (32\% of predicted), and voluntary peak cough flow was $100 \mathrm{~L} / \mathrm{min}$, revealing a severe restrictive syndrome. Two days later, supplemental $\mathrm{O}_{2}$ was discontinued and ventilatory mode was changed to volume-cycled (Elysee 150, ResMed, San Diego, California) (see Table 1) and $\mathrm{CO}_{2}$ values returned to normal. NIV was interrupted only for very short intervals for meals and hygiene, during which $\mathrm{CO}_{2}$ levels rapidly increased.

On the fourth day of continuous NIV, a level 3 nasal bridge sore $(\mathrm{NBS})^{3}$ that had been growing, decreased patient tolerance to NIV. Other models of interface were tried to improve tolerance; however, the patient did not tolerate any kind of nasal pillows and could not adapt to a mouthpiece with and without lip seal, due to lack of oral sensitivity and anxiety. She was then introduced to a total face mask model that, having no contact with the nose, immediately improved the patient's comfort and tolerance, maintaining the efficacy of continuous NIV support with no change in ventilatory settings. During the following 2 days, as the NBS healed, she was encouraged to pro- gressively use nasal pillows for daytime ventilation, and maintained the total face mask during sleep. The diagnosis of amyotrophic lateral sclerosis (ALS) (with no bulbar muscle impairment) was confirmed by electromyography. Before discharge, 8 days after admission, caregivers were trained in oximetry interpretation, ventilator and interface management, as well as manual and mechanically assisted coughing techniques.

At 4 month follow-up evaluation, no changes in the treatment strategy were necessary, since she continued to be 24-hour ventilator dependent, with intact bulbar muscle and $\mathrm{S}_{\mathrm{pO}_{2}}>95 \%$, so tracheostomy was not considered. ${ }^{15}$

\section{Case 2}

A 70-year-old male with ALS with bulbar muscle impairment (FVC equal to maximal insufflation capacity) $)^{16,17}$ had been for 13 months on NIV using a total of 16 hours/ day in a bi-level mode (VPAP IV ST, ResMed, San Diego, California) delivered through an oronasal mask. The patient was admitted to the hospital with respiratory-tract infection and respiratory acidosis. Continuous noninvasive ventilatory support was immediately initiated (see Table 1), resulting in correction of acidosis. Despite good adaptation to NIV, the chest showed weak expansion with low tidal volumes, even after adjustment of ventilatory settings. This fact was related to upper-airway obstruction due to bulbar muscle weakness and was corrected with neck hyperextension and mandibular protrusion. This maneuver may have facilitated glottis opening for better NIV efficacy, which became continuous from that point on.

Associated with respiratory infection the patient developed secretion encumbrance that could not be solved with mechanical in-exufflation (MI-E) (Cough-Assist, Philips Respironics, Murrysville, Pennsylvania) at optimal pressures, ${ }^{18,19}$ because of bulbar muscle weakness and inability to close the glottis. Secretion management was possible only with routine deep airway suctioning through the nose. After 24 hours of continuous NIV, a level $4 \mathrm{NBS}^{3}$ developed and a tracheostomy was proposed, due to incapacity to tolerate continuous NIV or maintain $\mathrm{S}_{\mathrm{pO}_{2}}>95 \%$, even with MI-E and suctioning. The interface was changed to the total face mask (with no change in ventilatory settings), involving no contact with the NBS. This provided comfortable and efficient ventilation support to the patient, giving him the time he needed to decide for tracheostomy.

\section{Case 3}

A 62-year-old male with ALS with bulbar muscle weakness, (FVC equal to maximal insufflation capacity) ${ }^{16,17}$ was on nocturnal ventilation support in bi-level mode (see Table 1) for 16 months. The patient was admitted to an intermediate care unit with the diagnosis of lobar pneu- 


\section{Continuous NiV Via Total Face Mask}

Table 1. Patient Characteristics, Nasal Bridge Sore Grade, and Ventilatory Settings

\begin{tabular}{|c|c|c|c|c|c|c|c|}
\hline Patient & Diagnosis & Age (y) & $\begin{array}{c}\text { Days of Continuous NIV } \\
\text { (oronasal mask) }\end{array}$ & $\begin{array}{l}\text { Nasal Bridge } \\
\text { Sore Grade }\end{array}$ & $\begin{array}{l}\text { Time With Total } \\
\text { Face Mask }\end{array}$ & $\begin{array}{l}\text { IPAP/EPAP } \\
\left(\mathrm{cm} \mathrm{H}_{2} \mathrm{O}\right)\end{array}$ & Ventilator settings \\
\hline 1 & ALS & 69 & 4 & 3 & $>4 \mathrm{mo}$ & $24 / 7$ & $\begin{array}{l}\text { IPAP/EPAP-24/7* } \\
\text { VT/PEEP-900/4 } \dagger\end{array}$ \\
\hline 2 & ALS & 70 & 1 & 4 & $1 \mathrm{~d}$ & $24 / 8$ & IPAP/EPAP-24/8* \\
\hline 3 & ALS & 62 & 7 & 4 & $7 d$ & $27 / 8$ & IPAP/EPAP-27/8* \\
\hline 4 & SCI & 44 & NA & NA & $11 \mathrm{~d}$ & NA & VT/PEEP-800/4† \\
\hline $\begin{array}{l}* \text { Bi-level } \\
\dagger \text { Volume } \\
\text { NIV }=\text { nc } \\
\text { IPAP }=\text { in } \\
\text { EPAP = } \\
\text { VT }=\text { tid } \\
\text { ALS }=\text { ar } \\
\text { NA }=\text { no } \\
\text { SCI }=\text { sp }\end{array}$ & $\begin{array}{l}\text { lation }\left(\mathrm{cm} \mathrm{H}_{2} \mathrm{O}\right. \\
\text { olled ventilatior } \\
\text { sive ventilation } \\
\text { ory positive air } \\
\text { tory positive ai } \\
\text { ume } \\
\text { ophic lateral scl } \\
\text { icable } \\
\text { ord injury }\end{array}$ & $\begin{array}{l}\text { /cm } \mathrm{H}_{2} \mathrm{O} \text { ) } \\
\text { pressure } \\
\text { pressure }\end{array}$ & & & & & \\
\hline
\end{tabular}

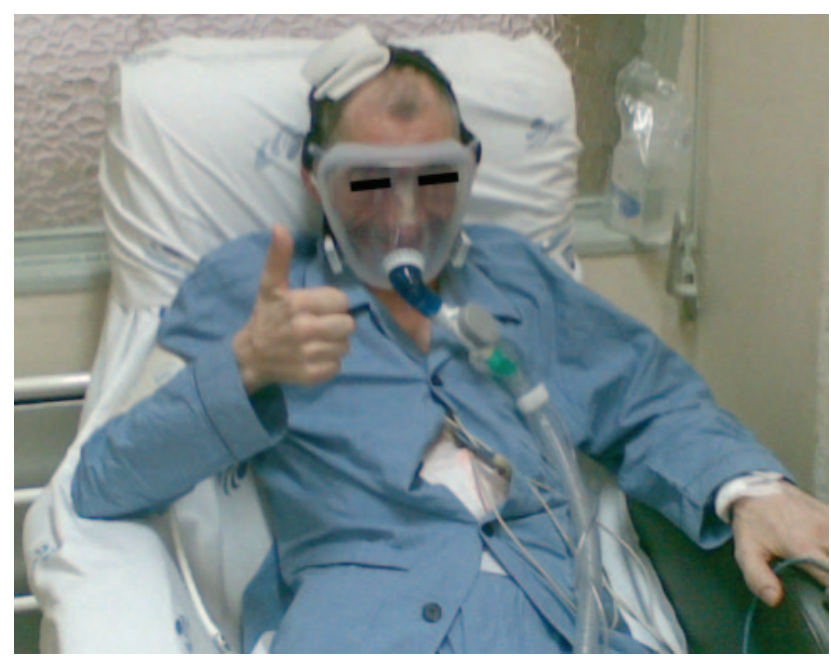

Fig. 2. Patient 3 with noninvasive ventilation through PerforMax total face mask in intermediate care setting. Wound care dressings over nasal bridge sore can be observed through the mask.

monia. In the previous weeks the bulbar muscles became weaker. To maintain his oxygen saturation above $95 \%$ he had been requiring increased periods of NIV during the day and more frequent MI-E sessions, so he had been proposed a tracheostomy. Continuous NIV use through a tight oronasal mask during that week resulted in a level 4 NBS. ${ }^{3}$ Adaptation to total face mask was immediate, and tolerance to continuous NIV was achieved for the following days (Fig. 2). Intensive chest physiotherapy and MI-E sessions were performed with success to help clear secretions and normalize $\mathrm{S}_{\mathrm{pO}_{2}}$. Given this good response, tracheostomy was postponed and the patient was discharged home 4 days later, though still on continuous NIV with total face mask and frequent MI-E with oximetry feedback. ${ }^{15}$ Unfortunately, due to NBS and rapid bulbar mus- cle weakening (with glottic impairment), frequent MI-E sessions were becoming hard to tolerate, and such a regimen became inefficient at home. The patient ultimately decided to accept tracheostomy, which was performed after a total of 7 days of continuous NIV delivered through total face mask.

\section{Case 4}

A 44-year-old female was the victim of an accidental 6 meters fall, resulting in several facial bone fractures and spinal cord contusion at the C5-C7 level. Four days later, in the context of nosocomial pneumonia, acute respiratory failure ensued, prompting intubation and invasive mechanical ventilation in the intensive care unit. The weaning process was difficult and prolonged. The first extubation attempt, on the third day of invasive mechanical ventilation, failed within 48 hours due to increasing hypoxemic respiratory failure and copious secretions. At the second extubation attempt, pre-extubation management was conventional other than for the aggressive use of MI-E via the tube as an alternative to airway suctioning. It was used up to every $10-15 \mathrm{~min}$, until $\mathrm{S}_{\mathrm{pO}_{2}}$ remained at $95 \%$ or greater in ambient air for at least 2 hours. ${ }^{20}$ Once this was the case, a ventilator weaning trial was attempted (60 min spontaneous breathing trial through a T-piece), and failed due to respiratory distress and exacerbation of hypercapnia. Since not yet consolidated facial fractures were a contraindication for the oronasal or nasal mask use, total face mask was employed as an alternative, allowing successful extubation after 9 days of invasive mechanical ventilation. Post-extubation MI-E at pressures of $40-60 \mathrm{~cm} \mathrm{H}_{2} \mathrm{O}$ to -40 to $-60 \mathrm{~cm}_{2} \mathrm{O}$ was performed as needed for full chest expansion, then complete chest emptying with abdominal thrust concomitant with exsufflation used as needed until $\mathrm{S}_{\mathrm{pO}_{2}}$ returned to $\geq 95 \%$. The patient was 
ventilated in volume mode through total face mask during the night and through mouthpiece during the day for 7 days, with an additional 4 days of nocturnal NIV only.

\section{Discussion}

The total face mask is an interface that seals around the perimeter of the face, where patients have smoother contours, overcoming some problems of other interfaces. It presents several desirable characteristics in an interface and can be used as an alternative to nasal or oronasal masks in case of NBS, claustrophobia, or to increase patient comfort. ${ }^{6}$ In the cases presented, a total face mask was successfully used to deliver NIV in a case of facial bone fractures and to overcome problems of NBS and discomfort caused by other interfaces. Compared with an older version of the total face mask, ${ }^{8,9}$ the PerforMax is smaller, therefore reducing dead space; the silicone contour is more easily adjustable to the patient's face and also has the advantage of flexibility to use with several ventilatory modes and parameters. Contrary to reports of the previous total face mask model use, $, 8,9$ in our work we included 24-hour ventilator dependent neuromuscular patients and patients ventilated in the intermediate care setting, without significantly increasing the nursing burden, due to the excellent adaptation of patients to the mask. No increase in $\mathrm{CO}_{2}$ levels or in breathing labor was registered with this model, and no patient needed endotracheal intubation.

In 3 of the patients presented here, NBS developed with variable speed, ranging from 24 hours to one week. Several measures can be used to prevent NBS, the most important being the reduction of headgear strap tension. ${ }^{6}$ This is often achieved in stable patients with mild respiratory failure, and especially with nocturnal NIV only. In situations of acute or acute-on-chronic respiratory failure, like the cases presented, this may not be as easily accomplished. Studies in acute patients treated with NIV showed an NBS rate of $13 \%{ }^{21}$ to $29 \% .{ }^{22}$ In order to most effectively reverse the changes in arterial blood gases, there may be a temptation to over-tighten the mask to minimize leakage, which can result in NBS, despite protection with wound-care dressings. Our first case refers to an undiagnosed ALS patient whose disease presented with acute respiratory failure. The lack of an interface allowing NIV use until stabilization of clinical condition and adaptation of the patient to the nasal prongs could have led to tracheal intubation and possibly tracheostomy to facilitate weaning afterwards..$^{23}$ The use of a total face mask was an effective solution to the NBS problem where other interfaces failed, while providing a much more comfortable ventilatory support. In the end, tracheostomy was not necessary and hospital stay was shortened.
Oropharyngeal dysmotility may have been the cause for reduced NIV efficacy on the second patient, since episodic lack of compliance seemed to be overcome by airway maneuver. Upper airway collapse has been associated with decreased tolerance and efficacy of both NIV and MI-E in ALS patients with severe bulbar muscle weakness ${ }^{24}$; however, there have been recent reports of effective MI-E sessions in this patient population. ${ }^{25}$ In our case series, both patients 2 and 3 had tolerated nocturnal or nocturnal plus daily periods of NIV for more than a year, and management with MI-E guided by oximetry. Progression of bulbar muscle weakness reduced MI-E sessions' efficacy in resolving secretion encumbrance, and the call for aggressive deep airway suctioning led to tracheostomy being proposed, which is consistent with the results of Bach et al. ${ }^{15}$ In both cases, total face mask was used to provide the patient with some time to decide for a tracheostomy.

The fourth case is a patient with spinal cord lesion presenting prolonged weaning from invasive mechanical ventilation. It is presumed that ventilator weaning is necessary before patients can be safely extubated. ${ }^{26}$ However, more recently, a successful extubation protocol that included continuous NIV and MI-E was described for 157 continuously ventilator dependent patients who could not pass spontaneous breathing trials before or after extubation. ${ }^{20}$ The importance of efficient MI-E sessions in this patient (who had no bulbar muscle impairment) is consistent with the described protocol. The problem of facial bone fractures in a patient who required long periods of NIV was overcome by the excellent adaptability of the total face mask, and sealing around the perimeter of the face, which permitted her extubation to NIV.

In a Criner et $\mathrm{al}^{8}$ report with the previous total face mask model, side effects like mild skin irritation due to improper padding and compression of the nose during expiration when the mask was over-tightened were observed, and, in the study by Roy et al, ${ }^{9}$ out of 10 patients, 6 complained of aerophagia and 5 had mask leaks, which led to tracheal intubation in 2. In our study, no patient reported expected side effects from this interface such as eye irritation or dryness, gastric distention, or claustrophobia, and no significant mask leaks were noted. Moreover, it did not obstruct the patient's field of vision, and allowed some degree of communication with the relatives and healthcare team. Although the case-series character of this work does not allow us to objectively compare NIV efficacy between total face mask and other masks in terms of leaks or alveolar ventilation, the facts that ventilatory parameters required no alteration after total face mask was applied, and that patients used it for several days without complications, suggest that efficacy was probably not decreased, as was demonstrated with the previous total face mask model. ${ }^{8,9}$ Total face mask use allowed earlier discharge of patients by avoiding intubation or permitting earlier extu- 


\section{Continuous NiV Via Total Face Mask}

bation with success, and warranted comfortable NIV support for ALS patients during decision making about tracheostomy. The concomitant use of MI-E when needed permitted overcoming the secretion encumbrance problem as long as glottic function was preserved.

We conclude that the PerforMax total face mask is a useful and effective interface in patients who do not tolerate a nasal or oronasal mask. It may offer more comfort and better healthcare to these patients.

\section{REFERENCES}

1. Wood KA, Lewis L, Von Harz B, Kollef MH. The use of noninvasive positive pressure ventilation in the emergency department: results of a randomized clinical trial. Chest 1998;113(5):1339-1346.

2. Conti G, Cavaliere F, Costa R, Craba A, Catarci S, Festa V, et al. Noninvasive positive-pressure ventilation with different interfaces in patients with respiratory failure after abdominal surgery: a matchedcontrol study. Respir Care 2007;52(11):1463-1471.

3. Gregoretti C, Confalonieri M, Navalesi P, Squadrone V, Frigerio P, Beltrame F, et al. Evaluation of patient skin breakdown and comfort with a new face mask for non-invasive ventilation: a multi-center study. Intensive Care Med 2002;28(3):278-284.

4. Mehta S, Hill NS. Noninvasive ventilation. Am J Respir Crit Care Med 2001;163(2):540-577.

5. Elliott MW. The interface: crucial for successful noninvasive ventilation. Eur Respir J 2004;23(1):7-8.

6. Nava $\mathrm{S}$, Navalesi $\mathrm{P}$, Gregoretti C. Interfaces and humidification for noninvasive mechanical ventilation. Respir Care 2009;54(1):71-84.

7. British Thoracic Society Standards of Care Committee. Non-invasive ventilation in acute respiratory failure. Thorax 2002;57(3):192211.

8. Criner GJ, Travaline JM, Brennan KJ, Kreimer DT. Efficacy of a new full face mask for noninvasive positive pressure ventilation. Chest 1994;106(4):1109-1115.

9. Roy B, Cordova FC, Travaline JM, D’Alonzo GE Jr, Criner GJ. Full face mask for noninvasive positive-pressure ventilation in patients with acute respiratory failure. J Am Osteopath Assoc 2007;107(4): 148-156.

10. Antonelli M, Conti G, Pelosi P, Gregoretti C, Pennisi MA, Costa R, et al. New treatment of acute hypoxemic respiratory failure: noninvasive pressure support ventilation delivered by helmet - a pilot controlled trial. Crit Care Med 2002;30(3):602-608.

11. Chiumello D, Pelosi P, Carlesso E, Severgnini P, Aspesi M, Gamberoni $\mathrm{C}$, et al. Noninvasive positive pressure ventilation delivered by helmet vs. standard face mask. Intensive Care Med 2003;29(10): 1671-1679.

12. Racca F, Appendini L, Berta G, Barberis L, Vittone F, Gregoretti C, et al. Helmet ventilation for acute respiratory failure and nasal skin breakdown in neuromuscular disorders. Anesth Analg 2009;109(1): 164-167.

13. Racca F, Appendini L, Gregoretti C, Stra E, Patessio A, Donner CF, et al. Effectiveness of mask and helmet interfaces to deliver noninvasive ventilation in a human model of resistive breathing. J Appl Physiol 2005;99(4):1262-1271.

14. Navalesi P, Costa R, Ceriana P, Carlucci A, Prinianakis G, Antonelli $\mathrm{M}$, et al. Non-invasive ventilation in chronic obstructive pulmonary disease patients: helmet versus facial mask. Intensive Care Med 2007;33(1):74-81.

15. Bach JR, Bianchi C, Aufiero E. Oximetry and indications for tracheotomy for amyotrophic lateral sclerosis. Chest 2004;126(5):15021507.

16. Kang SW, Bach JR. Maximum insufflation capacity. Chest 2000; 118(1):61-65.

17. Bach JR, Goncalves MR, Paez S, Winck JC, Leitao S, Abreu P. Expiratory flow maneuvers in patients with neuromuscular diseases. Am J Phys Med Rehabil 2006;85(2):105-111.

18. Sancho J, Servera E, Marin J, Vergara P, Belda FJ, Bach JR. Effect of lung mechanics on mechanically assisted flows and volumes. Am J Phys Med Rehabil 2004;83(9):698-703.

19. Winck JC, Goncalves MR, Lourenco C, Viana P, Almeida J, Bach JR. Effects of mechanical insufflation-exsufflation on respiratory parameters for patients with chronic airway secretion encumbrance. Chest 2004;126(3):774-780

20. Bach JR, Goncalves MR, Hamdani I, Winck JC. Extubation of patients with neuromuscular weakness: a new management paradigm. Chest 2010;137(5):1033-1039.

21. Meduri GU, Turner RE, Abou-Shala N, Wunderink R, Tolley E. Noninvasive positive pressure ventilation via face mask. First-line intervention in patients with acute hypercapnic and hypoxemic respiratory failure. Chest 1996;109(1):179-193.

22. Ferrer M, Esquinas A, Arancibia F, Bauer TT, Gonzalez G, Carrillo A, et al. Noninvasive ventilation during persistent weaning failure: a randomized controlled trial. Am J Respir Crit Care Med 2003;168(1): 70-76.

23. Bradley MD, Orrell RW, Clarke J, Davidson AC, Williams AJ, Kullmann DM, et al. Outcome of ventilatory support for acute respiratory failure in motor neurone disease. J Neurol Neurosurg Psychiatry 2002;72(6):752-756.

24. Sancho J, Servera E, Diaz J, Marin J. Efficacy of mechanical insufflation-exsufflation in medically stable patients with amyotrophic lateral sclerosis. Chest 2004;125(4):1400-1405.

25. Senent C, Golmard JL, Salachas F, Chiner E, Morelot-Panzini C, Meninger V, et al. A comparison of assisted cough techniques in stable patients with severe respiratory insufficiency due to amyotrophic lateral sclerosis. Amyotroph Lateral Scler 2011;12(1):26-32.

26. Harrop JS, Sharan AD, Scheid EH Jr, Vaccaro AR, Przybylski GJ. Tracheostomy placement in patients with complete cervical spinal cord injuries: American Spinal Injury Association Grade A. J Neurosurg 2004;100(1 Suppl Spine):20-23 . 